\title{
Extent-of-motion thresholds under subject-relative and object-relative conditions'
}

\author{
OLIVIA SHAFFER AND HANS WALLACH
}

SWARTHMORE COLLEGE

The experiment was designed to discover the threshold extent of motion at medium speeds amounting to 41,82, and $164 \mathrm{~min} . / \mathrm{sec}$., and to compare the perception of motion arising from subject-relative displacement with the perception of motion arising from object-relative displacement. Extent thresholds were found while velocity was kept constant. Different groups of ten $S$ s were used for each displacement velocity, and for each $S$ the extent threshold was twice obtained by the method of constant stimuli, once under subjectrelative and once under object-relative displacement conditions. Sensitivity to brief displacements of a continuously visible target was high; average thresholds ranged from 1.0 to $4.4 \mathrm{~min}$. under the various conditions employed. The thresholds were higher for subject-relative conditions and the slower displacement velocities and lower for objectrelative conditions and faster displacements.

By subject-relative displacement we mean displacement of the entire content of the visual field relative to $S$; by object-relative displacement we mean displacement of parts of the visual field relative to one another so that a pattern change occurs. Wallach (1959) defended this distinction on the grounds that (1) induced motion often occurs in the case of object-relative displacement-which object is seen as moving depends on pattern relationships rather than on objective motion; (2) the threshold for motion arising from object-relative displacement depends on pattern relationships ( $J$. F. Brown, 1931), and is probably generally lower than the threshold for subject-relative motion.

While it is a simple matter to present an object in subject-relative displacement only-a single moving object in an otherwise homogeneous visual field is the most convenient instance-object-relative displacement necessarily involves subject-relative displacement as well as object-relative displacement. Duncker (1929), nevertheless, succeeded in isolating motion perception based on object-relative displacement by using displacement velocities below the threshold for motion perception based on subject-relative displacement. He found that under these conditions objectively stationary objects would just as readily appear to move as objectively moving objects and that, when either a surrounded pattern or its surround was displaced at a rate below the subject-relative threshold, the surrounded pattern always appeared to move while the surround appeared immobile, no matter which of the two was moving objecti. $1 y$.

With motion perception apparently resulting from two different conditions of stimulation, namely subjectrelative and object-relative displacement, one should assume that the psychological processes thatlead to the corresponding motion experiences differ at least in part. However, the nature of motion experience itself does not depend on the type of motion stimulation; it is the same for subject-relative and for object-relative stimulation. Therefore, when we speak for the sake of brevity of subject-relative and object-relative motion, we do not imply any distinction in motion experience; the distinction rather lies in conditions of stimulation.

Leibowitz (1955) defends a similar but not identical distinction. He distinguishes motion discrimination based on "the magnitude of initial photochemical events" from motion discrimination based on "observation of change of position." Motion discrimination based on observation of change of position would be likely to enter most strongly into what is here called objectrelative motion, but according to Leibowitz' interpretation it can also enter into subject-relative motion if exposure time is long enough. The major independent variable distinguishing the two types of motion, according to Leibowitz, is exposure time: at very short exposure times, only initial photochemical events, which depend on speed, exposure time and brightness, are important, while for longer exposure times, any factor which facilitates perception of change of position, such as the existence of a framework, will influence motion perception. Leibowitz found that at a short exposure time (.25 sec.) the addition of a reference grid had no effect on velocity threshold, although the grid did have an effect at a longer exposure time (16 sec.). Leibowitz' condition without grid was not, however, entirely subject-relative: the edges of the motion field werevisible to $\mathrm{S}$.

Brown and Conklin (1954) have performed the only purely subject-relative velocity threshold experiment that we are aware of. They found a threshold of $18 \mathrm{~min} . / \mathrm{sec}$. at an exposure time of $.5 \mathrm{sec}$; this amounts to an extent of motion at threshold of $9 \mathrm{~min}$. With increasing exposure time, the threshold decreased to an apparently asymptotic value of $9 \mathrm{~min} . / \mathrm{sec}$, at 16 sec. exposure time (extent of motion at threshold: $144 \mathrm{~min}$.).

No previous experiment has found the extent-ofmotion threshold while keeping velocity constant as did our experiment. We expected that the object-relative extent threshold would be lower than the subjectrelative extent threshold for any given speed. In the case of the high speeds used by us, exposure time at threshold 
was very short so that, if Leibowitz were correct in asserting that the existence of a framework makes no difference at short exposure times, one would not expect any difference between the conditions in our experiment.

\section{METHOD}

\section{Apparafus and Stimuli}

With his left eye covered $\mathrm{S}$ was seated so that his right eye was within a few inches of a half-silvered mirror. The mirror, which was turned approximately $45^{\circ}$ to $S^{\prime}$ 's frontal plane, and the upper part of $S^{\prime} s$ body were surrounded by a box which contained openings through which light from the stimulus patterns could reach the mirror. All visual displacements were caused by a slight turning of the mirror. The mirror was mounted on a vertical rod which was connected (through gears) to a wide metal har extending horizontally and supported in two places to prevent its sagging; turning of the mirror was caused by movement of this bar. The extent of movement of the bar was controlled by a set of pins which confined its motion and whose positions could be varied. The speed of motion of the bar was controlled by a radial notch in the face of a solid wheel attached to a constant-speed motor. When the notch passed the rounded end of a rod which was attached to the end of the bar and was under spring tension, the end of the rod engaged the notch and the bar was carried along with the notch as far as the pins would allow. The extents of the visual displacement resulting from the bar movements as controlled by the various pin distances were determined by a computation which took the length of the bar, the gear ratio and the mirror law into account.

The stimulus for subject-relative motion was produced by placing a black paper pattern over a light box located to S's right. For half the Ss in each speed group the stimulus was a luminous outline square subtending $264 \mathrm{~min}$. of visual angle; it surrounded a luminous disk of $102 \mathrm{~min}$. visual angle. For the other half of the Ss, the disk was covered and only the square could be seen. The subject-relative stimulus was reflected in the mirror and became displaced when the mirror was turned, either to the left or to the right dependent on the wheel's rotation direction.

For object-relative motion, the square used in the subject-relative situation was the moving stimulus. It was seen to surround a disk subtending $184 \mathrm{~min}$. of visual angle. The disk was seen through the mirror and hence was never displaced. It was produced by a light box straight in front of $S$ so placed that the disk was for S optically centered in the outline square. The intensity of the subject-relative stimulus was so chosen that it was clearly visible yet did not illuminate the darkened experimental room. The "object-relative disk" was matched for apparent brightness when seen through the mirror.

\section{Subjects and Procedure}

Thirty undergraduates served as Ss, ten in each speed group. With half the Ss in each speed group the subject-relative threshold was measured first, while the other half started with the object-relative threshold. The displacement speeds were 41,82 , and 164 min./ sec. Prior to the threshold measurements, each S was given a preliminary ascending series under the objectrelative condition in order to acquire data on induced motion and also in order to give $S$ experience with the kind of motion experience to be reported. For this preliminary series, $\mathrm{S}$ was given one trial at each extent, starting with the smallest displacement until he first reported motion. Following the first motion report, $\mathrm{S}$ was given two or three further trials at the same extent and then, unless $S$ had reported correct motion directions for all trials at that extent, he was given trials at greater extents. The issue here was whether S saw the objectively displaced outline square or the stationary disk in motion, with the latter case amounting, of course, to induced motion.

Thresholds were measured by the method of constant stimuli. For each threshold, eight trials were given for each of seven extents, one of which was a no-displacement presentation. The extents were chosen on the basis of preliminary work using the method of limits with Ss not used in the experiment proper. The order of the stimuli was determined by using eight random permutations of the seven extents. The extents used for measuring the object-relative threshold in the low and medium speed groups were $0, .3, .8,1.3,1.8,2.3$ and $2.8 \mathrm{~min}$. For the same groups, the extents used for measuring the subject-relative threshold were $0, .8,1.8$, $2.8,3.8,4.8$ and $5.8 \mathrm{~min}$. In the high speed group, the extents used for measuring the object-relative threshold were $0, .3, .55, .8,1.3,1.8$ and $2.3 \mathrm{~min} .$, and the extents used for measuring the subject-relative threshold were $0, .8,1.3,1.8,2.8,3.8$ and $4.8 \mathrm{~min}$. Four Ss in the low speed group and one in the high speed group had subjectrelative thresholds higher than the greatest extent tested. These Ss' subject-relative thresholds were later tested using greater extents.

Displacement direction was left for half the trials and right for half the trials. For each trial, $S$ was asked to say whether he saw motion and if so whether it was left or right. In the object-relative situation he was also asked to state whether the square or the disk appeared to move. In the case of the object-relative condition $S$ was told that either the disk or the square might move left or right or they might both move in opposite directions. In the case of the subject-relative condition, $S$ was told that the whole stimulus pattern would move, left or right. A click could be heard when the mirror moved. S was told that motion would occur oniy with the click. A click was also heard on no-displacement trials. $S^{\prime}$ 's eyes were closed except during experimental trials. When $E$ was ready to start a trial, $S$ was asked to open his eyes. When $\mathbf{S}$ was ready to begin he said, "Ready." 
$\mathrm{S}$ was asked not to move his head after the ready signal. No fixation instructions were given. After each threshold determination, $\mathrm{S}$ was questioned about autokinetic motion and asked for comments.

\section{Induced Motion}

\section{RESULTS}

The first motion report in the preliminary objectrelative ascending series was analyzed for induced motion. It was assumed that this first report was the only one which could be said to be uninfluenced by set. The very first motion report was correct in direction for 27 of the $30 \mathrm{Ss}$. (Reports of disk motion were considered correct in direction when the direction given was opposite to the actual motion of the square.) For 23 of these $27 \mathrm{Ss}$, the first motion report occurred at a value below S's subject-relative threshold (three of the four exceptions were in the highest speed group). Fourteen of the 27 Ss reported the disk as moving in the first report. Since the square alone was objectively displaced, these reports may be considered instances of induced motion.

According to Duncker's findings all perceived motion that occurred at rates below the subject-relative motion threshold should have been induced motion, that is, motion of the disk. Instead we found that in nearly half the cases (11 out of 23) no induced motion occurred.

\section{Statistical Analysis of Thresholds}

For the purpose of comparing thresholds obtained under different conditions, each S's threshold for each condition was calculated separately. For each displacement extent the percentage of motion judgments correct in direction minus the percentage of motion judgments incorrect in direction was computed and marked on a graph, where the ordinate represented percentage of correct motion judgments and the abscissa displacement extents. An S's threshold was the extent at which the curve connecting these points crossed the $50 \%$ line. (In case a curve crossed the 50\% line twice, the threshold was found by redrawing the curve by connecting the midpoints of adjacent segments.) Since the distribution of thresholds appeared to be skewed, nonparametric tests were used.

The effects of certain factors, which were irrelevant to the major purposes of the experiment, were analyzed for the medium speed group. These factors were the presence or absence of the disk in the stimulus used for measuring the subject-relative threshold, the effect of test order, the difference between the first 28 and the second 28 judgments for both thresholds. None of these factors showed large or significant effects in the group analyzed, and they were ignored in the major analyses.

The effect of speed on the subject-relative threshold. Larger extents of displacement were required to perceive subject-relative motion at lower speeds. The media $_{n}$ subject-relative thresholds were $1.4 \mathrm{~min}$. in the high speed group (range $1.0 \mathrm{~min}$. to $7.3 \mathrm{~min}$.), $2.4 \mathrm{~min}$. in the medium speed group (range $1.6 \mathrm{~min}$. to $5.8 \mathrm{~min}$.) and $4.4 \mathrm{~min}$. in the low speed group (range $1.6 \mathrm{~min}$. to more than 10.3 min.). A Kruskall-Wallace analysis of variance on the ranks of the thresholds demonstrated that the three groups differed from each other at the .05 level $(H=7.76, d f=2)$. Mann-Whitney $U$ tests between pairs of groups demonstrated that the high and low groups differed from each other at the .02 level, twotailed ( $U=17, N=10$ and 10$)$, although adjacent groups did not differ significantly.

The effect of speed on the object-relative threshold. Object-relative extent thresholds did not vary significantly with speed. The median object-relative thresholds of all three groups were 1.0 or $1.1 \mathrm{~min}$. A KruskallWallace analysis of variance on the ranks of the thresholds revealed that the three groups did not differ from each other $(\mathrm{H}=1.36, \mathrm{df}=2)$. Mann-Whitney $\mathrm{U}$ was not significant when any pair of groups was compared. The range of object-relative thresholds among all $30 \mathrm{Ss}$ was from $.65 \mathrm{~min}$. to $2.8 \mathrm{~min}$.

The difference between subject-relative and objectrelative thresholds. At all speeds, larger extents were required to perceive subject-relative motion than to perceive object-relative motion. The subject-relative extent threshold was higher than the object-relative extent threshold for every one of the $30 \mathrm{Ss}$. This result is significant by sign tests for each group taken separately $(p<.01$, two-tailed).

The difference between the two thresholds decreased with increasing speed. The median difference was 2.9 $\mathrm{min}$. for the low speed group, $1.7 \mathrm{~min}$. for the medium speed group, .6 min for the high speed group. In order to test this interaction statistically, the difference between the two thresholds was calculated for each $\mathrm{S}$, and a Kruskall-Wallace analysis of variance was performed on the ranks of the differences. H reached the .05 level of statistical significance $(H=7,61$, $d f=2)$. MannWhitney $U$ tests revealed that the medium speed group differed from the high speed group $(U=24, N=10$ and 10 , $\mathrm{p}<.05)$ and the two extreme groups differed from each other $(\mathrm{U}=16.5, \mathrm{~N}=10$ and $10, \mathrm{p}<.02)$.

The difference found between subject-relative and object-relative motion detection was tested in a second way in order to rule out the possibilities that the apparent difference might be due either to a difference in the ease of detecting the direction of motion (rather than the ease of detecting existence of motion) or to some difference in the stringency of criteria for reporting motion. In order to do such a test each S's motion reports, regardless of direction, to an actual displacement (hit rate) were evaluated relative to his motion reports to no-displacement trials (false alarm rate). $1.8 \mathrm{~min}$. was chosen as an appropriate displacement extent at which to evaluate the hit rate. It was desired to avoid any assumptions as to the nature of the operating characteristic (the relationship between hit rates and false alarm rates). Norman (1964) has made this possible by showing that for certain combina- 
tions of false alarm rates and hit rates it is possible to say that performance in one situation is better than performance in another no matter what operating characteristic is assumed. For certain other combinations, the comparison is ambiguous; one must assume a particular shape for the operating characteristic before one can say which performance is better.

When subject-relative performance at $1.8 \mathrm{~min}$. displacement was thus compared with object-relative performance at $1.8 \mathrm{~min} .$, the results were as follows. ${ }^{2}$ In no case was subject-relative performance unambiguously superior to object-relative performance. In the high-speed group there were six ambiguous cases and four object-relative superior cases; in the medium speed group there were two ambiguous cases and eight object-relative superior cases; in the low speed group there were no ambiguous cases and ten object-relative superior cases. Considering ambiguous cases as ties, the superiority of object-relative performance is significant by sign tests at the .05 level in two groups, the medium and the low speed groups. The decrease in ambiguous cases with decreasing speed confirms the interaction between speed and type of motion reported above.

The correlation between subject-relative and objectrelative thresholds. In all groups there was a positive correlation between subject-relative and object-relative thresholds. A Spearman rank correlation coefficient was calculated for each group separately. The resulting correlations were .68 in the high speed group, .81 in the medium speed group, and .51 in the low speed group. The first two correlations are significant at the .05 level or better.

\section{General Characteristics of the Threshold Data}

Responses to no motion. There were a rather large number of motion responses on no-displacement trials (about 25\% for most groups and conditions). This relatively high false alarm rate may have been due in part to autokinetic motion.

Autokinetic motion reports. Every S reported occasional instances of obviously non-veridical motion, and some Ss were distinguished by frequent occurrence of non-veridical motion. Six of the 30 Ss reported that they frequently experienced slow smooth sideways motion prior to the occurrence of the click which marked mirror motion; these Ss said they normally gave motion reports only when a different motion was superimposed during the click. Some Ss appeared to develop autokinetic motion peculiar to the click: two Ss even reported short fast motions during a click heard when the motor was turned off and one $\mathrm{S}$ said he was able to see motion by imagining a click. Seven Ss frequently saw motion in two directions during the click that marked mirror motion; most of these Ss reported motion in the first direction seen unless the stimulus ended up in the place it started, in which case they reported no motion. Most Ss experienced up or down motion once or twice,
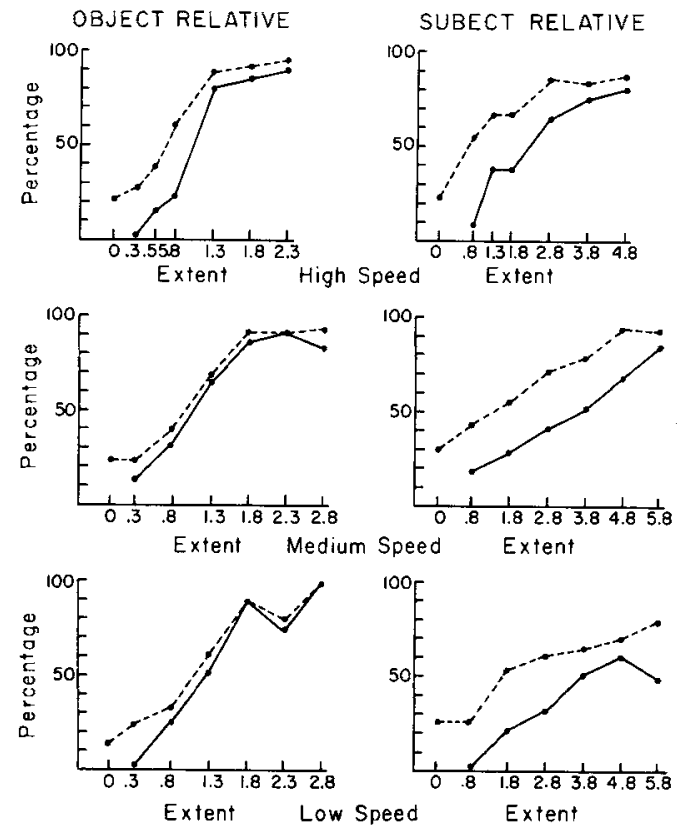

Fig. 1. Overall performance curves from each threshold determination. Each point represents 80 responses, 8 by each of $10 \mathrm{Ss}$. The broken curves represent percentage motion responses to each stimulus irrespective of direction, while the solid curves represent percentage motion reports correct in direction minus percentage motion reports incorrect in direction.

but none saw up or down motion frequently. Frequency of autokinetic motion reports was not greatly different for the subject-relative and the object-relative conditions. There was no apparent correlation between autokinetic reports and motion reports on no-displacement trials.

Direction errors. In the slow and medium speed groups, direction errors were rare in the objectrelative condition (31 errors or $5 \%$ of all motion responses on displacement trials), but more common in the subject-relative condition (114 errors or $19 \%$ of all motion responses). For these speed groups, the difference between the number of subject-relative direction errors and the number of object-relative direction errors was significant by sign tests $(p<.05)$. This difference may indicate that under subjectrelative conditions motion can be perceived without perception of its direction, or it might merely be a consequence of differences in the false alarm rate for the two types of motion. It is very difficult to distinguish between these alternatives.

Overall performance curves.

Given the marked individual differences in the thresholds, overall curves are not very meaningful. However, they are shown in Fig. 1 in order to give the reader a general idea of the overall results. For each speed and type of motion, the upper broken curve is the total percentage of motion reports at each extent, while the lower solid 
curve is the percentage of correct motion reports minus the percentage of incorrect motion reports. The first point of the broken curve is the false alarm rate. It will be noted that the tested displacement extents appear to cover a reasonable range in all cases except that of the subject-relative motion at the lowest speed. There, the greatest extent tested for all Ss (5.8 min.) showed only about $50 \%$ performance (correct minus wrong). Further testing of four Ss with larger extents suggested that the overall curve would not rise very much with extents up to $10.3 \mathrm{~min}$.

\section{SUMMARY AND DISCUSSION}

The major results were: (1) the median threshold extents of motion required to perceive subject-relative motion at speeds between $41 \mathrm{~min} . / \mathrm{sec}$. and $164 \mathrm{~min} . / \mathrm{sec}$. range from $4.4 \mathrm{~min}$. to $1.4 \mathrm{~min}$.; (2) at higher speeds, subject-relative motion can be perceived at smaller extents than at lower speeds; (3) object-relative motion can be seen at smaller extents than subject-relative motion (the object-relative extent threshold was about 1 min. at all speeds) but the difference between the two decreases with increasing speed; (4) induced motion occurs for extents below the subject-relative threshold, but not invaribly so, as should have been expected from Duncker's results.

The shortness of the time intervals occupied by the displacements used in obtaining the results has important implications. First, pursuit movements of the eye are not required for induced motion or for motion perception based on subject-relative displacement. The displacement for the first motion report, which was used in the analysis of induced motion, never in any case occupied more than .045 sec. $(1.8$ $\mathrm{min}$. at $41 \mathrm{~min} . / \mathrm{sec}$.). This is shorter than the reaction time for pursuit movements, which is in the order of $.200 \mathrm{sec}$. The subject-relative extent threshold was less than $4 \mathrm{~min}$. for the majority of Ss in the high and middle speed groups (17/20 Ss). This means that motions could be detected which took less than .05 sec. for completion. This means in turn that mere image displacement on the retina, without an accompanying eye movement or the existence of a framework, is a sufficient condition for the perception of motion.

The second implication of the short time intervals occupied by the displacements is the refutation of Leibowitz' hypothesis that the use of a framework should make no difference in motion perception at very short time intervals. Object-relative motion perception was shown to be superior to subject-relative motion perception for the $1.8 \mathrm{~min}$. extent. At this extent the displacement occupied an interval of .011 sec., .022 sec. and
$.044 \mathrm{sec}$. in the high, medium and low speed groups, respectively. These are much shorter times than the .1 sec. value below which Leibowitz (1955) suggests position change is irrelevant to motion perception. However, the interaction between speed and type of motion found in our experiment suggests that there may be a combination of some extremely short time interval (less than .01 sec.) with some high speed where the introduction of a framework would not have any effect.

No firm conclusions can be drawn from our results that subject-relative motion perception and objectrelative motion perception are basically different processes. The correlations found between the two thresholds appears to argue the opposite, namely, that there is a basic relationship between the two types of motion perception. But, with so many variables operating, this correlation may be interpreted in different ways. It could be due to the effect of a number of individual differences, e.g., visual acuity or attention. On the other hand, several further differences between the two types of motion perception were discovered: (1) object-relative motion is more easily detected than subject-relative motion; (2) subject-relative motion perception is more dependent on speed than objectrelative motion perception, which is mainly dependent on extent of displacement; (3) more direction errors are made in subject-relative motion judgments than in object-relative motion judgments.

\section{References}

Brown, J. F. The thresholds for visual movement. Psychol. Forsch. $1931,14,249-268$.

Brown, R. H., \& Conklin, J. E. The lower threshold of visible movement as a function of exposure time. Amer. J. Psychol., $1954,67,104-110$.

Duncker, K. Über induzierte Bewegung (ein Beitrag zur Theorie optisch, wahrgenommener Bewegung). Psychol. Forsch., 1929, $12,180-259$

Leibowitz, H. W. Effect of reference lines on the discrimination of movement. J. Opt, Soc. Amer., 1955, 45, 829-830.

Norman, D. A. A comparison of data obtained with different falsealarm rates. Psychol. Rev., 1964, 71, 243-246.

Wallach, H. Perception of motion. Scient. American, 1959, 201, 56-60.

\section{Notes}

1. The work was supported by Grant GB-2884 by the National Science Foundation to Swarthmore College, Hans Wallach principal investigator.

2. In the cases for which the false alarm rate was zero for both subject-relative and object-relative motion (three cases), performance in the condition with the higher hit rate was considered superior although strictly speaking such a comparison is ambiguous.

(Accepted for publication October 20, 1966.) 